\title{
Un approccio attivo alla geometria piana nella scuola primaria
}

\section{An active approach to plane geometry in primary school}

\author{
Ezio Scali \\ Insegnante-ricercatore scuola primaria - Torino, Italia \\ 凶ezioscali@inwind.it
}

"Le immagini parlano, o richiamano parole, perché non c'è immagine senza linguaggio"

Raymond Duval

Sunto / L'articolo descrive un'esperienza didattica realizzata in una classe quinta della scuola primaria: lo scopo dell'attività era di proporre una modalità di lavoro che permettesse agli alunni di attivare le loro potenzialità cognitive quando affrontavano situazioni geometriche nelle quali era loro richiesto di pianificare come si potessero calcolare le aree non note di figure geometriche. Tali situazioni sono spesso proposte in termini statici, attraverso lezioni frontali in cui il compito degli studenti è la fruizione. Le consegne hanno messo in evidenza quanto sia stretto il rapporto fra il linguaggio e gli aspetti iconici e figurali e come questo possa essere discusso con gli studenti. Mentre la discussione ha evidenziato diversi approcci ai significati coinvolti, confrontarsi con le situazioni proposte e interagire coi pari sembra aver aumentato la consapevolezza degli studenti circa il senso del calcolo delle aree.

Parole chiave: intuizione; visione; linguaggio; consapevolezza; rappresentazione.

\begin{abstract}
The article describes a teaching experience, carried out in a 5 th grade class of primary school. The aim of the activity was to propose a working method which could enable students to activate their cognitive potentialities when facing geometrical situations in which they were asked to plan how to calculate unknown areas of geometric figures.

Such situations are often proposed in static terms, through frontal lessons in which the students' task is to learn how to use formulas. The proposed tasks highlighted how tight is the relationship between the language and the iconic and figural aspects and how this can be object of discussion with the students. While the discussion highlighted several different approaches to the involved meanings, coping with the proposed situations and interacting with peers seems to have increased the students' awareness about the meaning of areas calculation rules.
\end{abstract}

Keywords: intuition; vision; language; awareness; representation. 


\section{Introduzione}

Un problema delicato e impegnativo per il lavoro dell'insegnante in ambito matematico è assumere realmente nella propria pratica didattica la complessità della costruzione del pensiero matematico, che è un

«processo lungo e progressivo nel quale concetti, abilità, competenze e atteggiamenti vengono ritrovati, intrecciati, consolidati e sviluppati a più riprese; è un processo che comporta anche difficoltà linguistiche e che richiede un'acquisizione graduale del linguaggio matematico».

(MIUR, 2012, p. 60)

Riflettere a fondo su questa affermazione delle Indicazioni Nazionali italiane del 2012 può permettere all'insegnante di costruire una progettazione che miri a perseguire per tutti gli allievi quegli aspetti di alfabetizzazione culturale che rappresentano ciò che un futuro cittadino deve possedere per compiere scelte consapevoli.

La matematica è parte di questa progettazione e la qualità dell'apprendimento può determinare la padronanza o meno di strumenti per leggere e interpretare la realtà e per orientarsi in essa. II tema della costruzione delle competenze ha assunto, in questa prospettiva, un'importanza cruciale, ma si ha l'impressione che si stenti, nella pratica didattica più diffusa, a comprendere i cambiamenti di sguardo che richiede il lavorare in un'ottica di costruzione di competenze: «cambiamenti non sostenuti, almeno in Italia, da un adeguato programma di formazione degli insegnanti» (Baccaglini Frank, Di Martino, Natalini \& Rosolini, 2018, pp. 92-93). Si assiste a una difficoltà a pensare che realmente si possa lavorare, in modo non episodico, mettendo al centro problemi «intesi come questioni autentiche e significative, legate alla vita quotidiana, e non solo esercizi a carattere ripetitivo o quesiti ai quali si risponde semplicemente ricordando una definizione o una regola» (MIUR, 2012, p. 62). Un aspetto di questa difficoltà è legato alla poca propensione a considerare la complessità della relazione educativa. La scuola ha sviluppato un notevole lessico rispetto ai "problemi legati alla vita quotidiana": problemi reali, problemi concreti, compiti autentici, compiti di realtà ecc. I libri di testo contengono pagine con questi titoli, ma affinché non restino solamente parole d'ordine alla moda, è necessario che si consideri il tessuto didattico, l'impianto complessivo che costituisce la globalità dell'esperienza scolastica vissuta dagli allievi. La letteratura offre numerosi esempi riguardo all'idea di successo scolastico (soprattutto in matematica) che si forma nella mente di un allievo, e di come questa idea non sia legata a ciò che realmente ha imparato o capito, ma ad altri fattori: al voto assegnato, alla fortuna, alla memoria, alla velocità. Le ricerche di Zan $(2007,2016)$ offrono molte riflessioni su queste problematiche e possono aiutare gli insegnanti a provare a scardinare pratiche didattiche che seguono per abitudine, senza la necessaria consapevolezza di ciò che si mette realmente in gioco.

\section{Uno sguardo alla geometria}

In questo articolo si prenderanno in considerazione alcuni aspetti dell'insegnamento della geometria piana nella scuola primaria, ${ }^{1}$ partendo dalla constatazione, costruita in molti anni di lavoro in classe, di quanto la geometria degli ultimi anni della scuola primaria possa costituire un serbatoio di appren-

1. La scuola primaria in Italia dura cinque anni e corrisponde alla scuola elementare nel Canton Ticino. 
dimenti illusori per i bambini. Un serbatoio attraente, perché spesso dà agli allievi l'impressione di aver "imparato" le figure geometriche, le loro caratteristiche, e le relative formule per calcolare misure significative; una trappola consolatoria anche per gli insegnanti, perché consente loro di offrire agli allievi un ventaglio di "successi" di gradazione diversa, in relazione al raggiungimento dell'obiettivo «Determinare I'area di rettangoli e triangoli e di altre figure [...]» (MIUR, 2012, p. 62): chi si arresta alla prima parte dell'obiettivo, chi si spinge appena più avanti, chi giunge a declinare tutte le formule. Ma le competenze acquisite sono nella sostanza veramente diverse? E che cosa le rende eventualmente diverse?

Viene da chiedersi se il lavoro geometrico sia svolto frequentemente in modo statico e avulso da una relazione con la realtà. Ma forse, prima ancora, tale lavoro non risulta in stretta relazione con il pensiero e con i modi in cui gli allievi vedono gli oggetti geometrici. Questa è un'esperienza che può sopprimere la motivazione e determinare un appiattimento del "fare", orientato verso un pensiero applicativo, strutturalmente riproduttivo.

Anche quando l'insegnante (o il libro di testo per lui) presenta agli allievi le formule per calcolare le aree delle figure geometriche, introducendole talvolta con una dimostrazione che tiene conto dei teoremi sottesi, i risultati di apprendimento non sono all'altezza delle attese.

Nel primo capitolo de Il pensiero produttivo, dedicato all'area del parallelogramma, Wertheimer (1997) offre una acuta analisi delle implicazioni inerenti alla possibilità per il bambino di giungere ad una comprensione profonda della struttura di quel problema. Allo stesso tempo chiarisce i limiti di un insegnamento che procede per dimostrazioni di teoremi attraverso lezioni frontali: la classe sa ripetere la formula e la dimostrazione che porta ad essa, ma le difficoltà rispetto alla proposta di lavorare sulla stessa figura orientata in modo diverso rende evidente che il problema di come si determina I'area di un parallelogramma non sia stato compreso in modo profondo.

L'insegnante descritto all'inizio del capitolo da Wertheimer (1997) è ancora oggi un buon esempio di insegnante soddisfatto del suo approccio all'insegnamento della geometria. II più delle volte non ha consapevolezza del fatto che si privano gli allievi di interessanti esperienze cognitive e culturali, che solo la scuola può offrire attraverso la mediazione dell'insegnante, in quanto è il luogo dell'incontro di un sapere disciplinare da costruire e del pensiero e del modo di vedere di ciascun allievo. In questo processo di apprendimento il confronto con il pensiero dei pari, cioè con gli altri pensieri e gli altri modi di vedere quel particolare lavoro, costituisce una ricchezza che si rischierebbe di perdere in una prospettiva orientata alla fruizione e all'applicazione.

Il compito dell'insegnante richiede dunque, in modo trasparente e rigoroso, la presa in carico del lavoro cognitivo che viene richiesto all'allievo e gli strumenti che egli ritiene legittimo utilizzare nella risoluzione di un qualsiasi problema, dove per strumenti non si intendono solo gli "attrezzi" del fare didattico, ma anche la possibilità interiore di esplorare, sperimentare, sbagliare. La consapevolezza dunque riguarda un approccio più generale al processo di insegnamento-apprendimento, perché, come sottolinea Pellerey, per la costruzione di competenze occorre «mettere in moto e orchestrare le proprie risorse interne, cognitive, affettive, volitive e utilizzare le risorse esterne in modo coerente e fecondo» (Pellerey, 2004, p. 12).

Numerosi studi in didattica della matematica (si vedano ad esempio Mariotti, 2005; Fischbein, 1993; Duval, 2018) hanno indagato in modo approfondito su molte problematiche riguardanti aspetti che vengono messi in gioco nelle situazioni didattiche di tipo geometrico: la distanza tra disegno e figura geometrica, il rapporto tra le proprietà figurali e i vincoli concettuali che caratterizzano il piano teorico del fare geometrico, il ruolo del vedere che orienta l'uso euristico delle figure geometriche nelle risoluzioni di problemi, il divario tra geometria e realtà. Alcune di queste problematiche verranno riprese in seguito. 


\section{Elementi generali di contesto}

In letteratura viene richiamata l'attenzione alla distinzione tra realtà e geometria (tra i tanti contributi, Laborde, 1988; Parzysz, 1988). La realtà non è trasferibile immediatamente nelle categorie geometriche. Così, se è indispensabile costruire e sostenere la consapevolezza che la geometria sia uno strumento per interpretare e modellizzare la realtà, nello stesso tempo è fondamentale essere prudenti nell'interpretare, ad esempio, ciò che gli allievi fanno per ragionare sulla forma del cortile della scuola e per trasferirla in una figura sul foglio, assumendo quest'ultimo momento come "geometrico". I problemi di natura epistemologica, psicologica e cognitiva presenti in questo passaggio vanno considerati con estrema attenzione al fine di non confondere i piani del discorso: la maturazione di un pensiero geometrico è un processo lungo e articolato che si fonda sull'acquisizione di una progressiva consapevolezza della relazione tra la visione, la figura, i concetti, il pensiero e la parola.

Da queste considerazioni è nata l'esigenza di mettere in gioco, su un piano didattico, con i bambini della scuola primaria, delle ipotesi di lavoro che, almeno in parte, rendessero dinamico l'approccio alle figure geometriche e la relazione di queste con le formule per determinarne l'area. Determinante, affinché ciò sia possibile, è il contratto didattico che si costruisce con i bambini, relativo a che cosa viene associato al "fare matematico". Questo aspetto è legato alla possibilità per l'allievo di pensare la matematica come un luogo di esperienze di ragionamento e di parola, dove l'accostamento alla disciplina avviene attraverso il progressivo passaggio da strumento ad oggetto degli elementi che via via si incontrano nel processo di apprendimento, e al successivo passaggio da oggetti a strumenti quando l'oggetto può divenire risorsa per ulteriori apprendimenti (Douady, 1986). Questo passaggio è reso possibile dall'uso, mediato dall'insegnante, di modalità di rappresentazione del pensiero e dell'azione di cui il bambino acquisisce gradualmente consapevolezza.

Nel progetto in cui si inserisce il lavoro della classe che verrà considerata in questo articolo, fin dall'inizio della scuola primaria viene curata la possibilità per il bambino di esprimere compiutamente il proprio pensiero, a cui viene data dignità di testo scritto attraverso la scelta didattica del prestamano: I'insegnante, per tutta la fase che precede la possibilità per il bambino di scrivere autonomamente, offre la sua mano per trascrivere il pensiero dell'allievo, mantenendone la ricchezza, talvolta sostenendone lo "sgrovigliamento", in qualche caso aiutando l'allievo a completare, arredare, definire frammenti di pensiero. Questa scelta ha origine dalla constatazione che, soprattutto per allievi che hanno un pensiero più articolato di quanto una relazione povera e deprivata con il linguaggio permetterebbe loro di esprimere, la mediazione dell'adulto è indispensabile per costruire la convinzione che la parola accompagna ogni azione, mentale e agita. La richiesta di "spiegare il ragionamento", frequente ormai anche nei libri di testo, senza una adeguata azione didattica che costruisca la consapevolezza di cos'è un ragionamento, si traduce sovente in scritture stereotipate e rituali.

La lingua che rende consapevoli ha una stretta relazione con un'altra scelta didattica di questo lavoro, quella di far esplorare i significati delle operazioni aritmetiche per un tempo lungo, all'interno di situazioni problematiche che il bambino possa cogliere come vere o verosimili, per poi giungere alla tecnica di calcolo scritto in un secondo momento, come organizzazione economica delle strategie spontanee, rispondente ai significati del numero fino a quel momento appresi. ${ }^{2}$ Questa cornice metodologica risponde anche all'esigenza di valorizzare l'idea che il pensiero matematico non sia soltanto applicazione di tecniche e regole fornite dall'esterno, costruendo in tal modo dei tasselli per lo «sviluppo di un atteggiamento positivo rispetto alla matematica» (MIUR, 2012, p. 61) perché le esperienze vissute hanno fatto intuire all'allievo «come gli strumenti matematici che ha imparato

2. Per eventuali approfondimenti, il riferimento è al Progetto "Bambini, maestri, realtà", DIMA-UniGe (Boero, 1990) e a Boero, Dapueto, Ferrari, Ferrero, Garuti, Lemut, Parenti e Scali (1995). 
ad utilizzare siano utili per operare nella realtà» (MIUR, 2012, p. 61). Questa competenza così importante, ma così complessa da pensare nella sua realizzazione nel tempo, appare come una guida ineludibile per il compito dell'insegnante.

\section{Il contesto specifico dell'esperienza}

L'esperienza che verrà descritta di seguito va contestualizzata, come si è detto in precedenza, in un'abitudine a ragionare sulle situazioni senza avere a disposizione preventivamente le regola, le procedure, le tecniche di calcolo scritte necessarie a risolverle. La costruzione graduale dell'idea che la matematica non è un insieme di nozioni, formule e tecniche da apprendere a memoria richiede che il docente, nel suo contratto didattico con la classe, costruisca anche la legittimità di poter riflettere liberamente sulla situazione oggetto di lavoro. Richiede in sostanza di lavorare sull'errore e sul ruolo che può avere nell'apprendimento e sulla valorizzazione reale del processo di pensiero rispetto alla produzione di un risultato.

Esplicitare, seppur in modo sintetico, la cornice in cui si inseriscono le esperienze presentate è necessario per avere elementi per comprenderne le potenzialità (e per riflettere criticamente anche sui limiti) che esse possono offrire a bambini che sempre di più, nella vita sociale, appaiono fruitori di esperienze, spesso virtuali, più che attori. Tuttavia, I'alunno può assumere un atteggiamento attivo solo se questo è la trama del tessuto quotidiano della pratica didattica.

L'esperienza è stata realizzata in una classe quinta, composta da 21 allievi, di una scuola primaria di Piossasco, un comune della seconda cintura di Torino. Alle attività che vengono di seguito descritte hanno partecipato 20 allievi (un allievo con disabilità grave non ha partecipato al lavoro). Nel corso delle classi terza e quarta era stato svolto un intenso lavoro geometrico a partire dall'osservazione del comportamento della luce del sole dapprima durante la giornata, poi nel corso dei mesi, e delle ombre che essa produceva in diverse situazioni. Gradualmente i bambini erano pervenuti ad utilizzare il punto di vista geometrico come una chiave privilegiata per interpretare i fenomeni osservati: ad esempio il fatto che a mezzogiorno l'ombra risultasse più corta di quanto fosse al mattino o nel pomeriggio, oppure la comprensione della rotazione, sul piano del cortile, della luce del sole passante per un foro praticato in un bastone sistemato in posizione verticale. La riflessione in classe sulle categorie geometriche aveva portato ad istituzionalizzare l'esistenza del "triangolo dell'ombra", cioè di quel triangolo che si forma considerando un oggetto, l'ombra che esso proietta e il raggio di sole che unisce in modo ideale le estremità dell'ombra e dell'oggetto. Questa modellizzazione non era di carattere statico, poiché era legata al movimento apparente del sole nel tempo della giornata e la riflessione che essa consentiva riguardo ai fenomeni studiati ne determinava il carattere di modello interpretativo. I bambini hanno partecipato in modo vivace a questa esperienza di approccio alla relazione tra esperienza fisica, esperienza di osservazione e sviluppo del pensiero argomentato in chiave interpretativa, dove il rapporto tra il vedere e il vedere con la mente era uno dei fulcri della possibilità di ricostruire mentalmente le relazioni che all'occhio non era dato di cogliere.

Accennare a questa esperienza permette da un lato di identificare passaggi importanti nel sapere costruito in classe a proposito del senso della geometria, e dall'altro di rintracciare le motivazioni della possibilità di far lavorare gli allievi su consegne che richiedevano un approccio di tipo dinamico anche per lo studio delle figure geometriche.

Durante la classe quarta, con i bambini erano state definite le formule riguardanti il perimetro e I'area di triangoli e rettangoli, che divenivano così strumenti per l'accesso alla possibilità di ragionare su nuove figure. Nel corso della prima parte della classe quinta si è deciso perciò di proporre l'esperienza didattica oggetto di questo lavoro: la costruzione delle formule per il calcolo delle aree 
dei parallelogrammi, dei rombi e dei trapezi.

Le consegne date richiedevano agli allievi di ragionare sulla figura presentata di volta in volta, disegnata su un foglio, con la possibilità di orientarla soggettivamente. Generalmente gli allievi iniziavano posizionando il disegno della figura in una posizione prototipica, ma sovente il foglio veniva ruotato per esplorare la figura da posizioni diverse. Inoltre, non veniva richiesta né la misurazione, né la scrittura dei calcoli, né il raggiungimento di un risultato numerico che definisse l'area. Per ogni tipo di figura sono state svolte tre lezioni della durata media di un'ora e mezza l'una. Le tre lezioni riguardavano: la risoluzione del problema, la discussione di bilancio delle soluzioni selezionate dall'insegnante, e un'attività di argomentazione e di istituzionalizzazione delle formule costruite. II percorso complessivo ha avuto la durata di dieci lezioni (compresa quella finale di assegnazione di item INVALSI). II presupposto pedagogico era che anche nelle situazioni presentate tradizionalmente in modo statico potesse essere efficace proporre e suscitare uno sguardo attivo ed esplorativo, che consentisse al bambino di mettere in gioco sia gli aspetti figurali della situazione, sia quelli concettuali e che questo richiedesse di separare l'attività mentale sulle figure dalla misura e dal calcolo.

Dal punto di vista dell'insegnante è stato importante monitorare ciò che succedeva per capire come queste consegne raggiungessero gli allievi, tutti gli allievi. Questo ha richiesto di prestare attenzione a molti focus di osservazione.

Un primo focus riguardava dove avvenisse il lavoro del bambino, se all'interno della figura o all'esterno, e con quali modalità. Si è trattato di osservare se l'allievo intravedesse la possibilità di manipolare e modificare la figura oppure intendesse mantenerla intatta concentrando il pensiero nella ricerca di forme compensative. Una chiave di lettura è rappresentata dai contributi di Fischbein (1992; 1993), che hanno messo in luce la necessità di pensare ai concetti figurali, come sintesi di due aspetti centrali del fare geometrico: I'aspetto figurale e l'aspetto concettuale, non sovrapponibili, ma nello stesso tempo fortemente intrecciati. «Secondo questa prospettiva, quando ci si riferisce alle figure geometriche si possono considerare tre categorie: la definizione, l'immagine (basata sull'esperienza percettiva-sensoriale, come l'immagine di un disegno) e il concetto figurale» (Sabena, Ferri, Martignone \& Robotti, 2019, p. 130).

Un secondo aspetto riguardava se e come il bambino modificasse il proprio atteggiamento nel susseguirsi delle proposte di lavoro oppure se queste venivano affrontate con modalità fisse perché rassicuranti dato che erano già state sperimentate con successo in situazioni precedenti.

Un terzo focus concerneva l'osservazione di come l'intreccio tra la cognizione individuale e le modalità di confronto e di ragionamento collettivo (nel piccolo gruppo e nelle discussioni di classe) permettessero la maturazione della possibilità di accedere a un pensiero razionale consapevole sostenuto anche dal piano di lavoro operativo sulle figure.

Infine, si è posta l'attenzione sul ruolo del linguaggio e sulla sua interazione con l'aspetto figurale. Monitorare in classe questo aspetto non è stato semplice e l'osservazione su questo piano è stata necessariamente limitata: in molti casi si sono potute fare solo inferenze sul corso del pensiero del bambino osservando il trattamento della figura e la sua corrispondenza con le tracce scritte del ragionamento seguito.

A questo proposito i contributi di Duval (2018) sulla visione aiutano a penetrare le caratteristiche del "vedere spontaneo" e del "vedere geometrico" e del rapporto di questi con il pensiero verbale. Secondo Duval, due principi governano la visualizzazione nella geometria elementare. II primo riguarda il fatto che il riconoscimento visuale di forme si fa in opposizione ad altre forme possibili che restano sullo sfondo, secondo le due modalità del riconoscimento per giustapposizione o per sovrapposizione. II secondo afferma che qualsiasi contorno di una configurazione è scomponibile in diversi contorni chiusi e che questi possono essere ricombinati per ottenere una configurazione diversa da quella originaria. Nella geometria elementare questi «sono i due approcci euristici fondamentali per la risoluzione di problemi» (Duval, 2018, p. 219). C'è uno stretto rapporto cognitivo tra le immagini e il linguaggio, o per meglio dire dello sguardo, del dire e del dire a sé stessi. Continu- 
iamo con le parole di Duval:

«Qualsiasi riconoscimento iconico implica un riconoscimento discorsivo, che abbiamo chiamato verbalizzazione silenziosa. [...] In questo senso, il linguaggio non segue l'azione, ma l'accompagna come suo controllo interno. [...] Quasi sempre quando parliamo di verbalizzazione, ci riferiamo alla verbalizzazione orale, vale a dire esplicita. Questa verbalizzazione spontanea, che riprende la verbalizzazione silenziosa, come ha mostrato Vygotskij, è tanto per sé stessi quanto per chi ascolta, o per colui al quale sembra rivolgersi. Ė una verbalizzazione orale après coup, a posteriori, successiva, per nominare, o caratterizzare, nel contesto di uno scambio o di una comunicazione, l'attività che è stata appena compiuta».

(Duval, 2018, p. 226)

Questa lunga citazione da Duval chiarisce bene la complessità dell'attività sul piano iconico e cognitivo contenuta nell'attività che è stata richiesta, ma nello stesso tempo sollecita l'insegnante a non perdere occasioni come quelle di mettere in gioco proprio questo rapporto ricco e fecondo tra l'immagine, il pensiero e la parola orientato ad uno scopo.

\section{Prima consegna: l'area del parallelogramma}

Ai bambini viene consegnato il disegno di un parallelogramma su un foglio bianco. In precedenza, le caratteristiche relative alla figura (parallelismo e uguaglianza delle misure dei lati opposti) erano state esplorate collettivamente.

La consegna è: «Come si può trovare l'area di questa figura?». L'accento è messo sul come, viene ricordato che non si devono eseguire calcoli e non si deve giungere a un risultato. Ai bambini viene chiesto di lavorare individualmente, scrivendo con molta cura tutti i loro pensieri: questa prassi è normale nella classe e in genere i bambini non hanno bisogno che l'insegnante lo ricordi. Negli anni, si è cercato di sviluppare nei bambini il gusto di spiegare, di argomentare il perché delle decisioni ed essi sanno, perché anche questa è abitudine consolidata, che poi verrà effettuato un momento di confronto dei ragionamenti prodotti e una discussione collettiva che permetta di giungere a delle conclusioni concordate e a una sintesi provvisoria.

Un necessario inciso nell'analisi a priori: la funzione della consegna è quella di attivare le conoscenze pregresse in vista di un loro riutilizzo per risolvere un nuovo compito. Le conoscenze pregresse riguardano la possibilità di utilizzare le figure base (triangolo e rettangolo) per scomporre le nuove figure. Tuttavia, la consegna ha un carattere di apertura che può permettere altre strategie non canoniche, a volte meno efficaci ed economiche, ma pur sempre accettabili e corrette. La relazione fra conoscenze pregresse, nuova figura e pensiero del bambino si inserisce in una cornice in cui agiscono il ruolo dell'insegnante (scelta consapevole della consegna), gli aspetti matematici (le conoscenze possedute) e le possibilità del bambino, sul piano cognitivo ed emotivo, di passare dal piano dell'osservare a quello del fare utilizzando consapevolmente il saper fare e, sullo sfondo, il poter fare.

\subsection{Risultati della prima consegna}

Alcuni allievi scelgono autonomamente di riprodurre la figura anche sul foglio quadrettato.

Tutti i 19 allievi presenti concludono il lavoro giungendo a una soluzione, quattro di essi con la mediazione dell'insegnante. Le soluzioni si possono raggruppare secondo tre diverse modalità, che vengono elencate di seguito: 
Soluzione 1A. Tre bambini costruiscono un rettangolo mediante l'aggiunta di altri elementi che eliminino i "disturbi", cioè le parti sporgenti a destra e a sinistra (Figura 1). Uno di essi scrive: «lo trasformo il parallelogramma in un rettangolo aggiungendo due triangoli ai lati per rendere i lati perpendicolari».

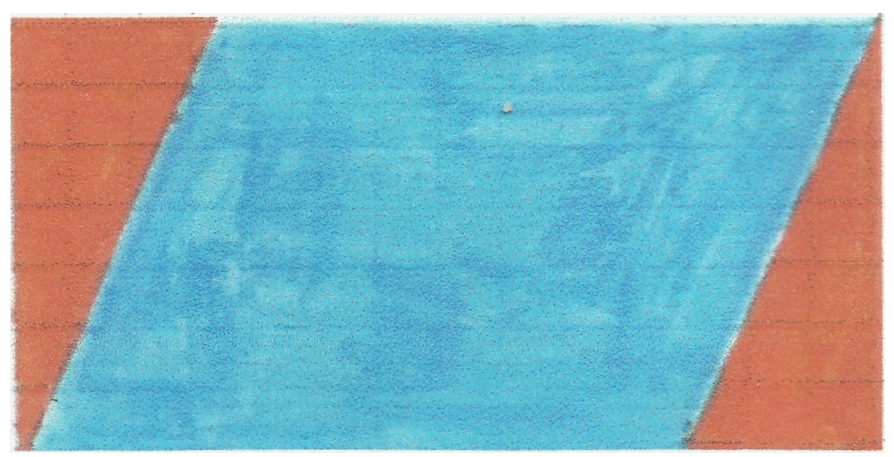

Figura 1. Riproduzione del disegno del parallelogramma realizzato dai bambini.

La figura viene così trasformata in un elemento conosciuto, il rettangolo, agendo dall'esterno forse per regolarizzare la figura presentata, con cui si fa fatica ad interagire così com'è. L'irregolarità sembra essere data dall'assenza di relazioni perpendicolari tra i lati che formano il contorno: da qui la necessità di racchiuderla in un rettangolo. Questi allievi procedono poi con sicurezza: dicono che si dovrà calcolare l'area del rettangolo ottenuto da cui si dovranno togliere le aree dei due triangoli aggiunti.

Soluzione 1B. Quattro allievi procedono per scomposizione interna della figura. Tre di essi tracciano una diagonale e dividono il parallelogramma in due triangoli (Figura 2).

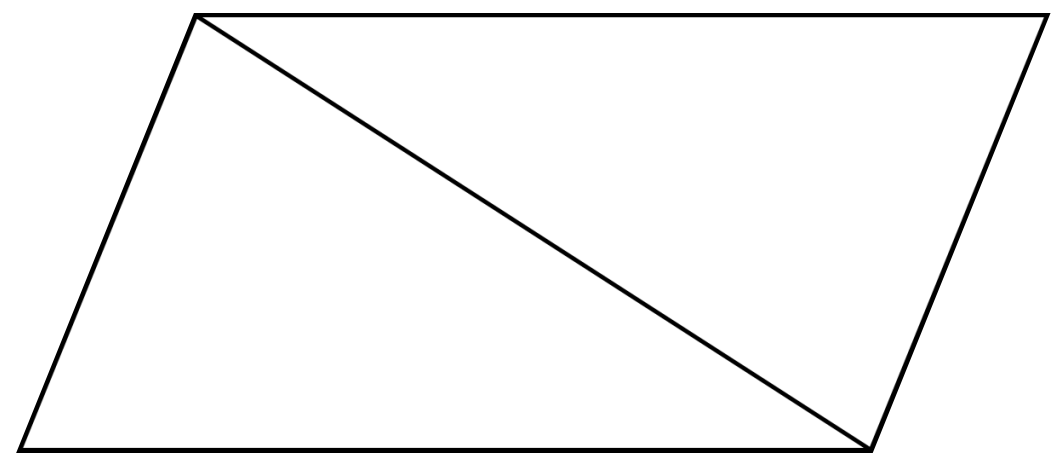

Figura 2. Riproduzione del disegno realizzato dai bambini.

Due di questi allievi ritagliano la figura lungo la diagonale per verificare se i due triangoli sono congruenti attraverso la sovrapposizione. Uno di essi scrive: «Mi sembravano uguali, ma non ero sicuro, perché nella mente li dovevo capovolgere, ma non era facile, mi confondevo. Allora ho tagliato e poi ho girato uno dei triangoli e sono riuscito a metterlo proprio sopra all'altro». ${ }^{3}$ Un terzo allievo giunge alla conclusione che sono congruenti per via argomentativa. II suo protocollo è interessante perché sembra inizialmente distaccarsi dall'aspetto figurale per costruire argomentazioni, su un piano linguistico-concettuale, ritornando poi al figurale come per un bisogno di coerenza tra i due aspetti:

3. Per tutti gli elaborati degli allievi è stato mantenuto il testo originale, errori ortografici e grammaticali compresi. 
«Ho disegnato la diagonale e ho diviso il parallelogramma in due triangoli. Mi sembrano uguali ma a vederli così non so, perché sono in due posizioni diverse. Però ho pensato: hanno un lato in comune, che è la diagonale, poi hanno anche gli altri due lati, sono lunghi uguale. Allora sono due triangoli con i lati uguali. Se immagino di far combaciare questi lati mi sembra di vederli uguali, devono essere uguali!».

La determinazione della congruenza dei triangoli non produce automaticamente delle conseguenze sul piano operativo: solo uno dei tre bambini afferma che si può calcolare l'area di uno dei triangoli e raddoppiarla per trovare I'area del parallelogramma. Nessuno dei tre ricava l'idea che, nel trovare I'area di un triangolo, il prodotto del lato per la relativa altezza possa poi non essere dimezzato, secondo il ragionamento che dividere per due e moltiplicare poi per due (dato che l'area del triangolo andrebbe poi raddoppiata per trovare quella del parallelogramma) sono due operazioni una inversa dell'altra. Sembrerebbe cioè che non necessariamente da intuizioni generate su un piano prevalentemente figurale possa originarsi un pensiero sul piano simbolico e concettuale. Tuttavia, si potrebbe ipotizzare che il piano dell'intuizione possa costituire il terreno fecondo su cui innestare un'elaborazione argomentata.

Una quarta allieva traccia le due diagonali formando quattro triangoli.

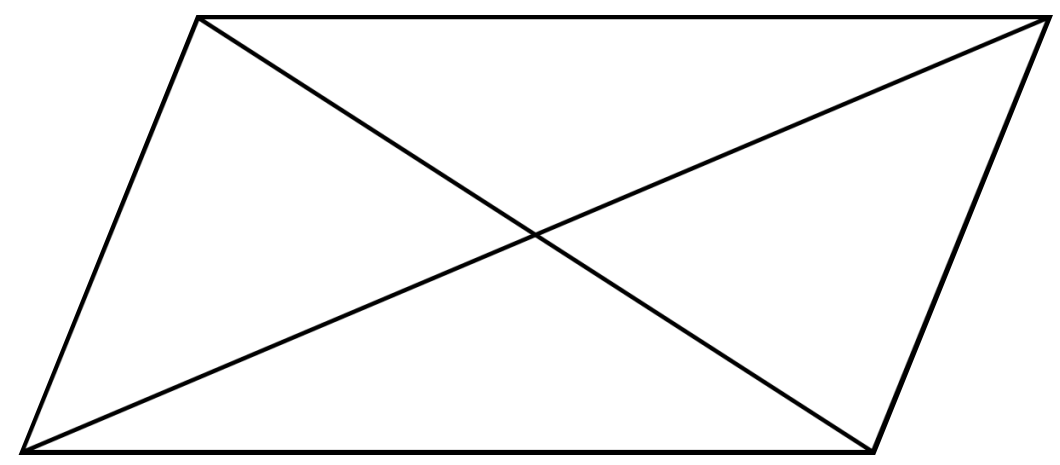

Figura 3. Disegno delle due diagonali con scomposizione del parallelogramma in quattro triangoli.

Non si pone il problema della congruenza a due a due dei triangoli e sostiene di dover calcolare le loro aree e poi sommarle.

Soluzione 1C. Dodici allievi trasformano il parallelogramma in un rettangolo senza modificarne la superficie. Tre di essi utilizzano il disegno mostrando come il triangolo rettangolo ottenuto tracciando I'altezza interna alla figura possa essere traslato e affiancato al lato opposto della figura generando così un rettangolo (Figura 4). Altri sei allievi eseguono lo stesso procedimento attraverso il ritaglio del triangolo e la ricomposizione del rettangolo. Due allievi seguono la stessa strategia di ragionamento esprimendola a parole, senza disegno o ritaglio. Anche in questi casi la soluzione praticata sembrerebbe rispondere al bisogno di eliminare il "disturbo" costituito dalla sporgenza a sinistra per ricreare una situazione di equilibrio. 


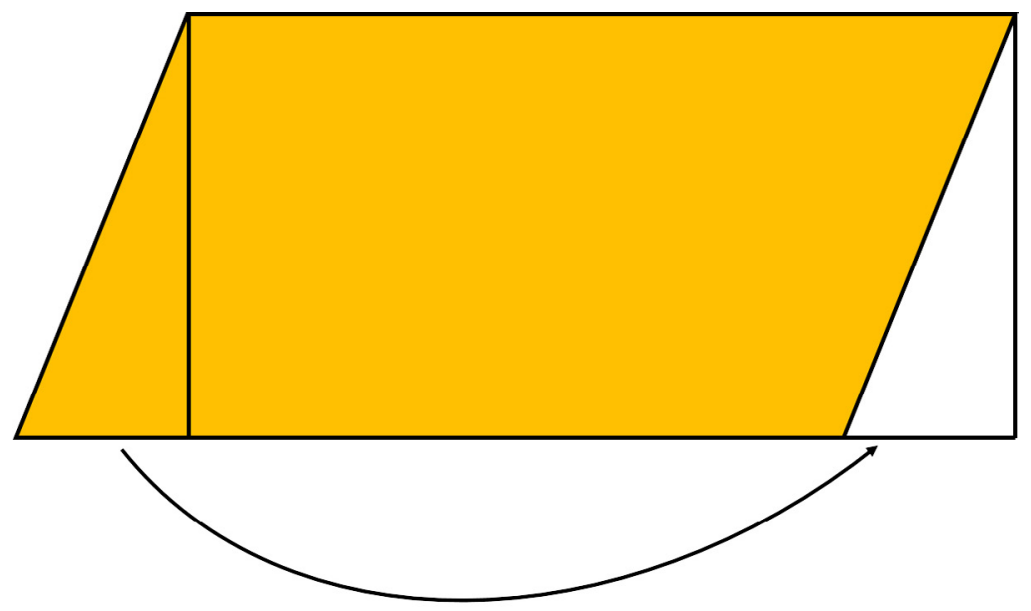

Figura 4. Trasformazione del parallelogramma in un rettangolo.

Un allievo, infine, taglia a metà il parallelogramma con una linea perpendicolare a una coppia di lati paralleli in modo da ottenere due trapezi rettangoli congruenti e ricompone la figura formando anch'egli un rettangolo.

In quest'ultimo gruppo di ragionamenti sembra prevalere uno sguardo che vede la possibilità di manipolare la figura ricorrendo alla somiglianza di caratteristiche con il rettangolo (una bambina, in modo espressivo scrive: «ll parallelogramma mi sembra un rettangolo dove uno si è appogiato e si è storto come è successo a un mobile nella mia cameretta»).

Si potrebbe dire che, in modo intuitivo, questi allievi percepiscono la proprietà di equiestensione tra parallelogramma e rettangolo. A questo proposito Fischbein osserva, facendo riferimento al già citato esempio di Wertheimer sul parallelogramma, che:

"Un bambino che ha capito che il parallelogramma può essere trasformato in un rettangolo (per esempio, usando le forbici) sembra acquisire una comprensione genuina dell'equiestensione tra le due aree [...]. Infatti, non c'è contraddizione tra il capire la soluzione significativa da un punto di vista del comportamento: "togliere a sinistra e aggiungere a destra" e seguire i passi della logica della dimostrazione analitica. Quando raggiunge o impara una dimostrazione formale, I'allievo non deve abbandonare la via strutturale della comprensione».

(Fischbein, 1992, pp. 14-15)

\subsection{Riflessioni sulle soluzioni emerse}

Situazioni come queste scaturite dalla prima consegna sollecitano riflessioni circa il ruolo e l'importanza dell'intuizione nella costruzione della conoscenza. Ancora Fischbein $(1992$, p. 7) nota come «l'intuizione è simultaneamente una forma derivata di cognizione - come lo è il pensiero - ed un programma per l'azione - come una percezione». L'intuizione si distingue dalla percezione perché è "soprattutto una forma di "interpretazione", una "soluzione ad un problema" » e nello stesso tempo si distingue dal pensiero perché «non è analitica, non è discorsiva, ma piuttosto una forma compatta di conoscenza» che non richiede una giustificazione estrinseca. Così, «nella sua forma anticipatoria, I'intuizione offre una prospettiva globale di una possibile soluzione del problema e, così, ispira e dirige i passi della ricerca e della costruzione della soluzione» (Fischbein, 1992, p. 7).

L'intuizione non richiede di per sé un linguaggio articolato e argomentato. I bambini che hanno scelto di scomporre il parallelogramma in due triangoli e i bambini che hanno scelto di formare il rettangolo (soluzioni $1 \mathrm{~B}$ e $1 \mathrm{C}$ ) hanno imboccato una strada che potrebbe condurre ad un pensiero formale. Ma 
è necessario dar parola al pensiero, che potrebbe legittimamente restare implicito, per far maturare la zona di sviluppo prossimale degli allievi (nel senso di Vygotskij, 1934/1990) rispetto alla capacità di esprimersi. Questo richiede una mediazione attenta da parte dell'insegnante nel proporre alla classe i passi successivi di meta-riflessione. A titolo di esempio si riporta un breve stralcio della discussione orchestrata dall'insegnante (indicato con I.) sul confronto tra le strategie di tipo 1A e 1C. La discussione collettiva, come altre, è stata registrata perché potesse essere riascoltata successivamente, in un'ottica di analisi a posteriori.

1. M1.: «Sì, tutti fanno un rettangolo, è uguale».

2. F.: «No! Perché... perché i rettangoli non sono uguali».

(...)

5. A.: «Uno è uguale al parallelogramma».

6. M2.: «Ha la stessa area, non è uguale».

7. M1.: «Però i triangoli sono uguali».

8. M2.: «Ma vedi che il rettangolo di S. è più grande di quello di R. perché R. è come se togliesse un pezzo dal rettangolo e poi lo mette dall'altra parte... Non cambia la figura è sempre un rettangolo, ma cambia la sua area...».

$(\ldots)$

16. I.: «Poco fa M2. diceva che il rettangolo ottenuto da S. ha una superficie maggiore del rettangolo ottenuto da R....».

17. V.: "Tu vedi che la base del rettangolo di R. è come la base del parallelogramma, solo che un pezzo l'abbiamo spostato...».

18. M2.: "Sì e la base dell'altro rettangolo [quello di S.] è più lunga perché c'è quella del parallelogrammo e poi quelle dei triangoli».

19. M1.: «Ah, mi è venuta un'idea, ma allora un parallelogramma è uguale a un rettangolo».

(...)

25. S.: «Sì sì, è un rettangolo e si calcola l'area nello stesso modo... perché... perché anche qui facciamo la base per l'altezza».

26. C.: "Questa volta sì, ho capito... Allora, la base del rettangolo è come la base del parallelogramma, è proprio uguale e anche l'altezza è sempre quella... è la stessa».

27. V.: "Allora non è che tutte le volte facciamo il disegno di R.... questa cosa che anche se disegni un altro parallelogramma diverso, più allungato è la stessa cosa...».

28. G.: "Succede così tutte le volte... cioè quella cosa che ha fatto R. si può fare sempre, in tutti i parallelogrammi».

$(\ldots)$

39. M3.: «Ma allora si vede che abbiamo trovato una specie di regola... I'area del parallelogramma si fa moltiplicando la base per l'altezza...».

40. V.: «Sì. Il parallelogramma è come un rettangolo, puoi spostare sempre un triangolo per farlo diventare un rettangolo».

Le parti in corsivo indicano come nella discussione i bambini pervengano a livelli di generalità, attraverso un distanziamento dall'esperienza svolta, ma mantenendola come riferimento.

I bambini discutono poi a lungo il ragionamento di tipo 1B (purtroppo, per un disguido tecnico manca la registrazione di questa parte) finché un allievo conclude che anche questo ragionamento permette di trovare la stessa regola per stabilire l'area di un parallelogramma. 


\section{Seconda consegna: l'area del rombo}

Tre settimane dopo viene proposta alla classe la consegna di trovare un modo per determinare l'area del rombo. Viene consegnata ai bambini una scheda su cui è riportata la figura. Anche in questo caso I'attività viene svolta individualmente.

\subsection{Risultati della seconda consegna}

Anche in questa occasione i bambini ragionano secondo strategie diverse.

Soluzione 2A. G., un bambino che nel caso del parallelogramma aveva risolto il problema con la soluzione 1A, continua a pensare l'area come una cornice che contiene la figura (Figura 5). II riempimento, dall'esterno, mediante il disegno del rettangolo che tocca tutti i vertici del rombo gli offre evidentemente una sicurezza.

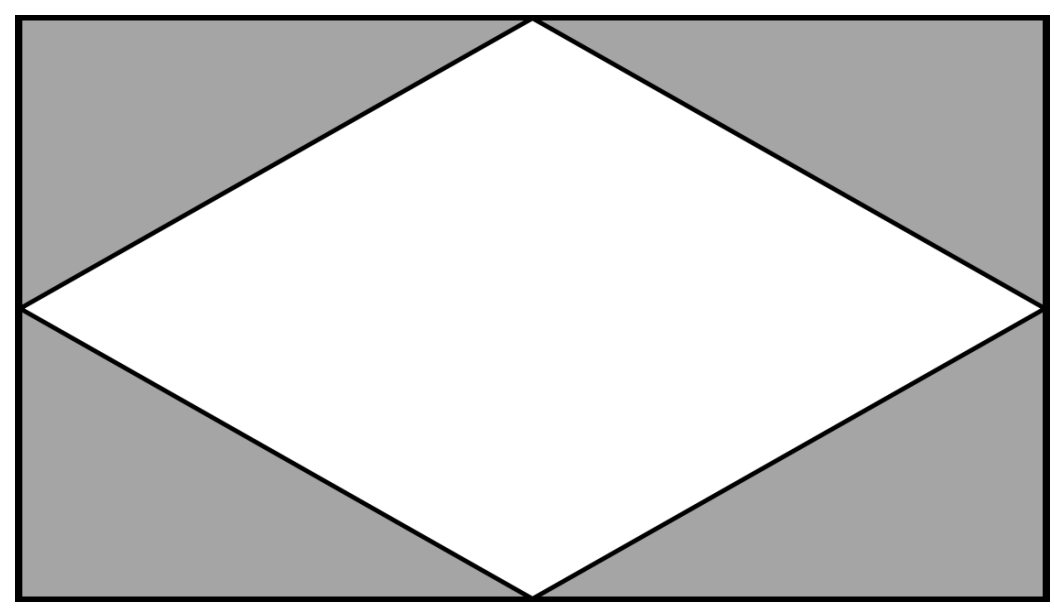

Figura 5. II rettangolo che "contiene" il rombo.

Nella verbalizzazione di G., si può intravedere la traccia di questo bisogno: «Nel rettangolo così il rombo ci sta tutto, poi posso togliere quello che è fuori del rombo». Sarebbe interessante indagare su un possibile bisogno di contenimento sottostante all'utilizzo ripetuto di strategie come queste, dove I'aspetto chiave, oltre al contenimento, sembra essere la difficoltà a "ritoccare" la figura. La metafora che accompagna questo tipo di atteggiamento, che compare se gli allievi sono legittimati a produrre un pensiero senza il condizionamento del giudizio, è quella dello scultore che dal blocco di materiale grezzo scava e toglie fino a raggiungere la forma progettata. A differenza dello scultore questo allievo progetta la "materia grezza" da togliere, anziché la forma.

Altri due bambini eseguono una strategia simile.

Soluzione $2 B$. Dodici bambini utilizzano le diagonali per ragionare all'interno del rombo. I loro sguardi, tuttavia, vedono aspetti diversi. A titolo di esempio, analizziamo le due soluzioni proposte da P. e da M2.

P.: «Divido il rombo in due triangoli con una diagonale e traccio le altezze, posso moltiplicare base per altezza poi dividere il risultato per due. Così trovo l'area di un triangolo, poi l'altro è uguale e unisco le misure» (Figura 6). 


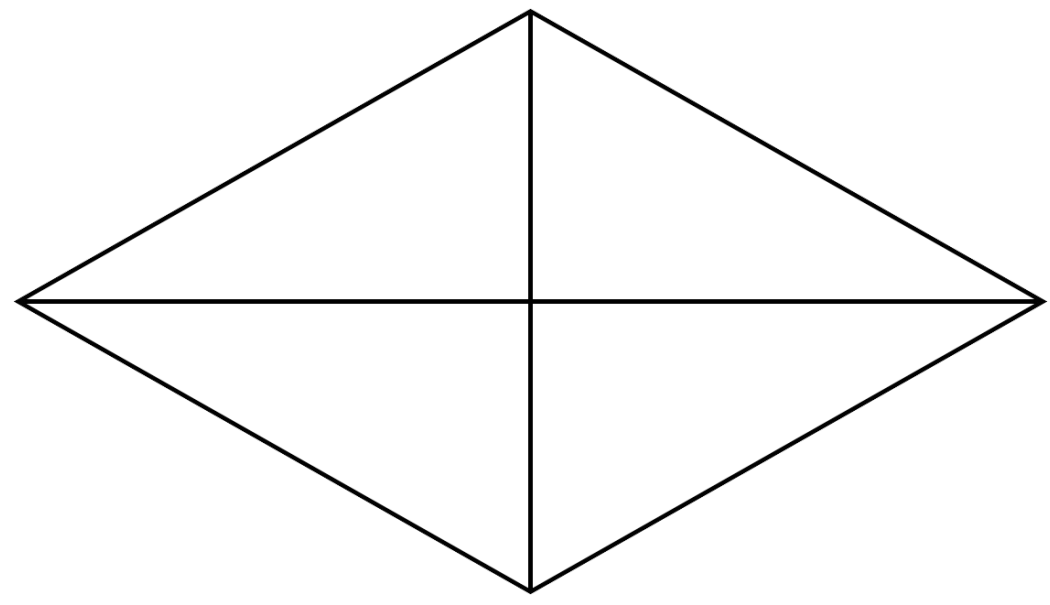

Figura 6. La strategia di P.: dividere il rombo in due triangoli congruenti.

Nella situazione precedente, P. era l'allieva che aveva suddiviso il parallelogramma in quattro triangoli, con l'idea di sommare poi le loro aree (soluzione 1B, Figura 3). Questa volta, tuttavia, percepisce la congruenza dei due triangoli. In entrambe le situazioni utilizza una strategia procedurale, anche questa rassicurante, lavorando all'interno della figura, secondo le modalità del piastrellista che "copre" tutta la superficie. Dal punto di vista dell'analisi a posteriori, ci si deve interrogare circa i possibili ostacoli che possono frapporsi all'accesso ad un pensiero analitico, dati dalla staticità (e dall'idea della replicabilità) di procedimenti come questi, in cui gli elementi disciplinari si intrecciano con modalità convergenti e chiuse relative alla risoluzione di problemi.

P., come pure G., la cui soluzione è stata considerata al punto precedente, hanno in comune una storia di acquisizione di consapevolezza a piccoli passi della possibilità di pensarsi capaci di affrontare situazioni problematiche, accettando il rischio di sbagliare. L'approccio attivo costruito trasversalmente in classe rispetto all'esperienza di apprendimento, attraverso una mediazione continua degli insegnanti, li ha aiutati a gestire il tempo interiore, condizione che ha permesso loro di poter recuperare conoscenze e abilità possedute. II terreno (scolastico) della geometria è un terreno di sfida, perché, a differenza di altri ambiti, come ad esempio la comprensione della lettura, può consentire atteggiamenti riproduttivi se una strategia viene individuata come accettabile in più situazioni.

M2. lavora sulla figura, parlando tra sé. Poi scrive:

«Devo disegnare la diagonale più corta, poi disegno I'altezza di uno dei due triangoli che vengono fuori. Ho visto che l'altezza è la metà della diagonale più lunga. Poi dovrei calcolare l'area del triangolo, ma non la divido per due perché è come se la metà inutilizzata [del rombo] andasse a formare un rettangolo se la mettiamo insieme al triangolo un po' da una parte e un po' dall'altra. Potrei dire che l'area del rombo si trova moltiplicando la diagonale per la metà dell'altra diagonale» (Figura 7). 


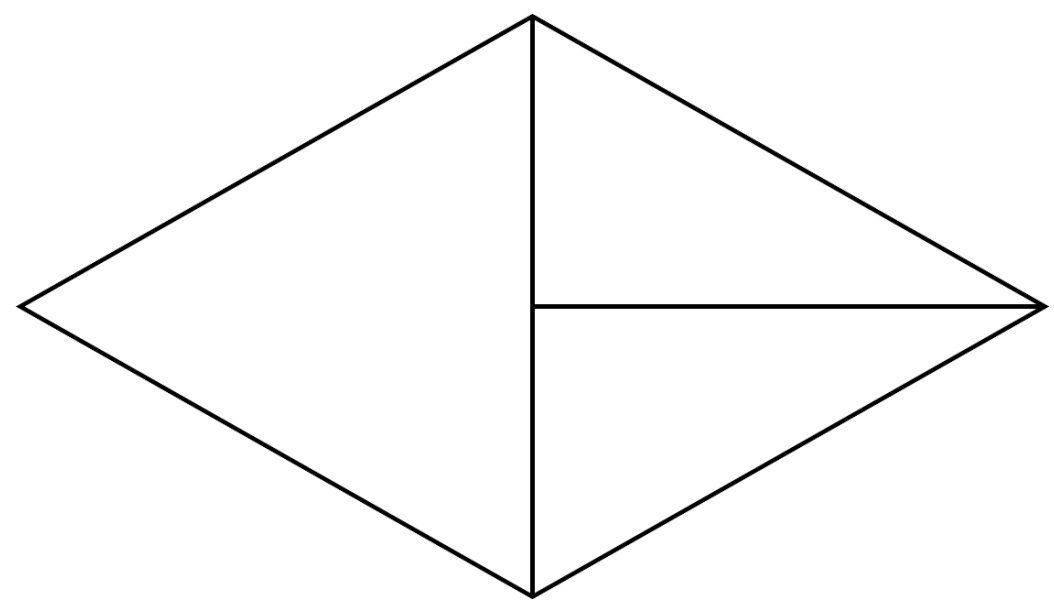

Figura 7. Rappresentazione del ragionamento di M2.

Questo protocollo mostra il passaggio dall'aspetto figurale a quello analitico-concettuale, con il trasferimento del lessico specifico dal rombo al triangolo costruito per poi reinterpretare dal punto di vista del rombo la situazione disegnata.

M3. offre altri due esempi interessanti di riflessioni che intrecciano in modo molto stretto il figurale e il concettuale, giustificando in modo molto convincente, l'idea di concetto figurale.

«Per trovare l'area del rombo provo a moltiplicare la base per l'altezza, cioè l'altezza sarà la diagonale minore e la base la diagonale maggiore. Sul disegno le traccio, ma la base attraverserà I'altezza, ma è giusto così. lo penso che sia giusto perché è come trovare l'area dei due triangoli e poi sommarle» (Figura 8 )

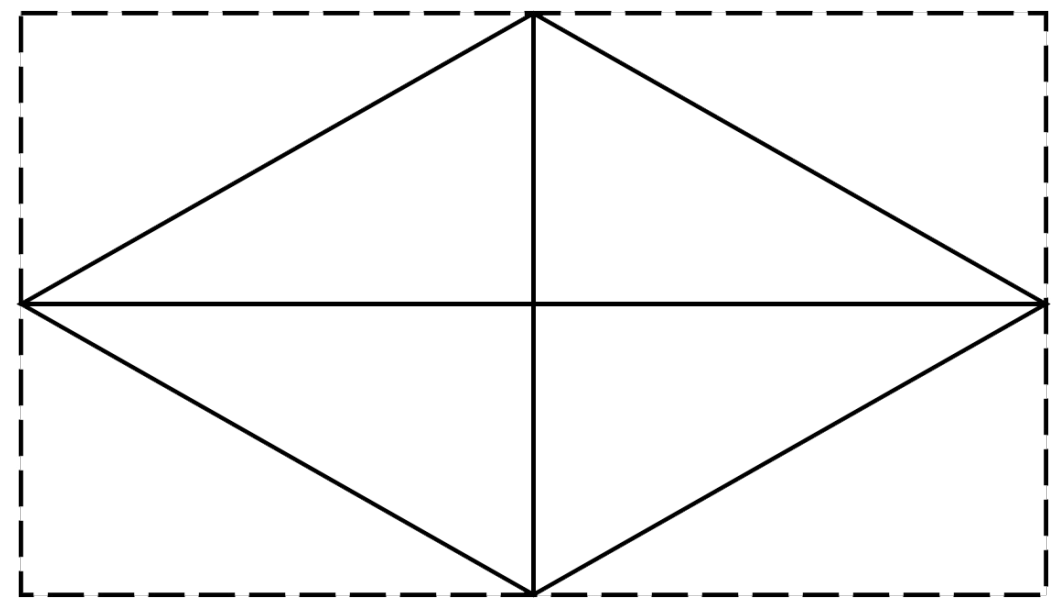

Figura 8. Prima rappresentazione di M3.

M3. sembra aver concluso il suo lavoro, ma non stacca gli occhi dal disegno. Sembra indecisa, prende un foglio e disegna il rombo con le diagonali, lo ritaglia e poi taglia lungo la diagonale minore:

«Ho provato a trasformare il rombo in un rettangolo, ho diviso a metà un triangolo e capovolgendo i due pezzi dall'altra parte in modo da fare un rettangolo mi sono accorta che il rettangolo che 
viene fuori deve essere la metà di quello che dicevo prima. Se moltiplico diagonale per diagonale formo un rettangolo che a il doppio dell'area del rombo. Allora quell'area la devo dividere a metà. La vera regola è diagonale per diagonale diviso 2» (Figura 9).

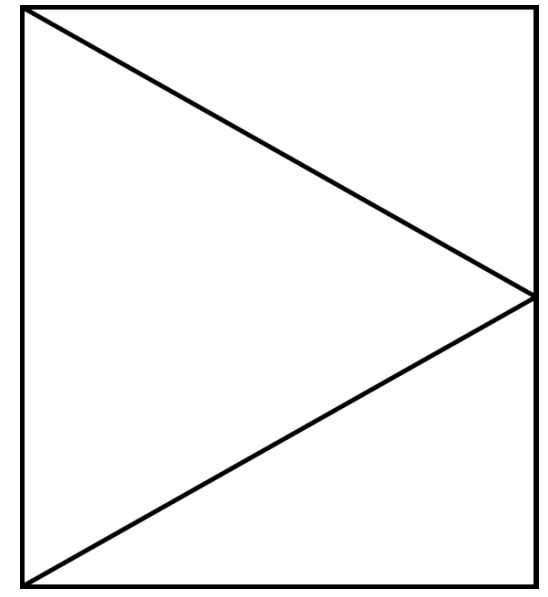

Figura 9. Seconda rappresentazione di M3.

Soluzione 2C. Come M3., anche C. inizia con la rappresentazione in Figura 8. Prende in mano la scheda dove ha eseguito la rappresentazione e la guarda a lungo, la rigira più volte. Ė molto concentrata, cancella le linee disegnate, prende il righello e la squadretta e traccia una linea.

«Un'idea che mi è venuta e di prendere questo rombo come se fosse un parallelogramma, tracciandone l'altezza e facendo base per altezza. Però stavo pensando che se semplicemente faccio un'altezza collegando l'angolo ottuso con l'altro angolo ottuso è più semplice».

C. traccia la diagonale seguendo la sua idea (la linea tratteggiata di Figura 10), osserva il disegno, poi riprende la matita e aggiunge: «No seguo l'idea di prima perché questa che stavo facendo non può essere un'altezza non è perpendicolare al lato».

C., come altri quattro compagni, pensa al rombo come un parallelogramma (traccia un'altezza del parallelogramma in Figura 10). La possibilità di orientarne la posizione (data dal foglio non incollato) e, soprattutto, la legittimità (costruita su un piano più generale di quello strettamente geometrico) di poter guardare la figura da più punti di vista agevola l'attivazione di un vedere che consente una nuova interpretazione.

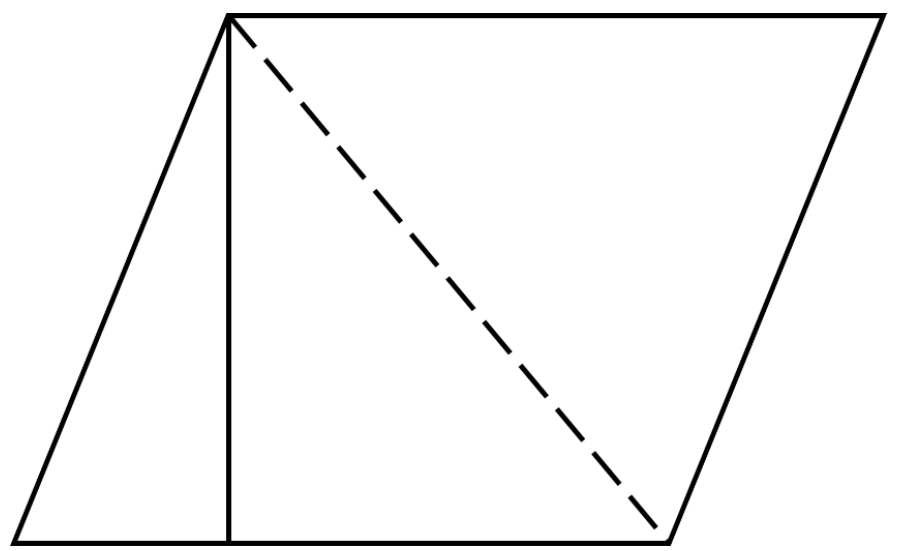

Figura 10. Seconda rappresentazione di C. 


\subsection{Riflessioni sulle soluzioni emerse}

Entrambe le allieve M3. e C. (soluzioni 2B e 2C) compiono almeno due operazioni interessanti rispetto al gioco delle ipotesi che esse producono.

La prima è I'andirivieni dal piano figurale a quello riflessivo concettuale: I'ipotesi formulata su un piano principalmente figurale (C. che interpreta il rombo come parallelogramma, recuperandone evidentemente le caratteristiche peculiari di lati opposti paralleli) o concettuale (M3. che interpreta la figura come formata da due triangoli scivolando subito sul piano procedurale del "cosa fare") viene verificata utilizzando l'altro piano (rispettivamente concettuale per C. e figurale per M3.), come per un'esigenza di coerenza tra i due aspetti. Così C. si pone il problema dell'altezza, giustificando attraverso il recupero di ciò che sa - l'altezza deve essere perpendicolare alla base - l'inesattezza del nuovo tentativo e M3. ricostruisce il vero rettangolo formato dal rombo sospendendo il pensiero analitico e operando sul piano figurale attraverso il ritaglio e la ricomposizione della figura.

La seconda operazione è la possibilità di ritornare indietro da una congettura formulata. Questa possibilità non avviene solo sul piano del lavoro geometrico perché si scopre l'inadeguatezza dell'ipotesi, ma prima ancora risiede nella possibilità interiore di vivere l'errore come un elemento naturale mentre si risolve un problema: colpisce il fatto che le due allieve non cancellino con tratti di penna la parte di testo errata, ma usino le parole (ad esempio, C. scrive: «No seguo l'idea di prima...») per completare il pensiero che comprende anche la parte da rigettare. Risulta importante che in situazioni come queste il piano della misura e del calcolo venga separato dalla risoluzione del problema, affinché il piano del ragionamento possa muoversi senza essere ostacolato dalla ricerca del risultato. Questo favorisce la possibilità di intrecciare il vedere la figura con il pensiero sulla figura o il pensiero mentre si opera sulla figura.

Anche questa fase si conclude con la progettazione da parte dell'insegnante di una discussione sul confronto tra le scelte risolutive che erano state riportate su una scheda. La riflessione giunge ad ammettere l'esistenza di due regole per calcolare l'area del rombo, a seconda che lo si trasformi in un rettangolo o che lo si pensi come un parallelogramma.

\section{7}

Terza consegna: l'area del trapezio

Qualche settimana dopo viene proposto alla classe il problema di trovare un modo per calcolare I'area del trapezio. La figura era stata oggetto di esplorazione nei giorni precedenti e, come nelle precedenti consegne, viene data agli allievi una scheda su cui è riportato il disegno di un trapezio scaleno.

In termini di analisi a priori ci si potrebbe attendere che il ragionamento di scomposizione interna della figura prevalga in accordo con la possibilità di vedere la forma come la giustapposizione di rettangoli e triangoli in conseguenza delle conclusioni emerse dalle attività precedenti.

\subsection{Risultati della terza consegna}

In effetti, tutti gli allievi, tranne uno (due allievi sono assenti), procedono per scomposizione.

Soluzione 3A. Dodici allievi individuano un rettangolo e due triangoli, con due soluzioni differenti riguardo a questi ultimi: alcuni uniscono i due triangoli laterali formando un unico triangolo, sulla base dell'identità della loro altezza (Figura 11a), altri mantengono i due triangoli rettangoli separati (Figura 11b). In entrambe le soluzioni si tratta di sommare le aree delle figure individuate. 

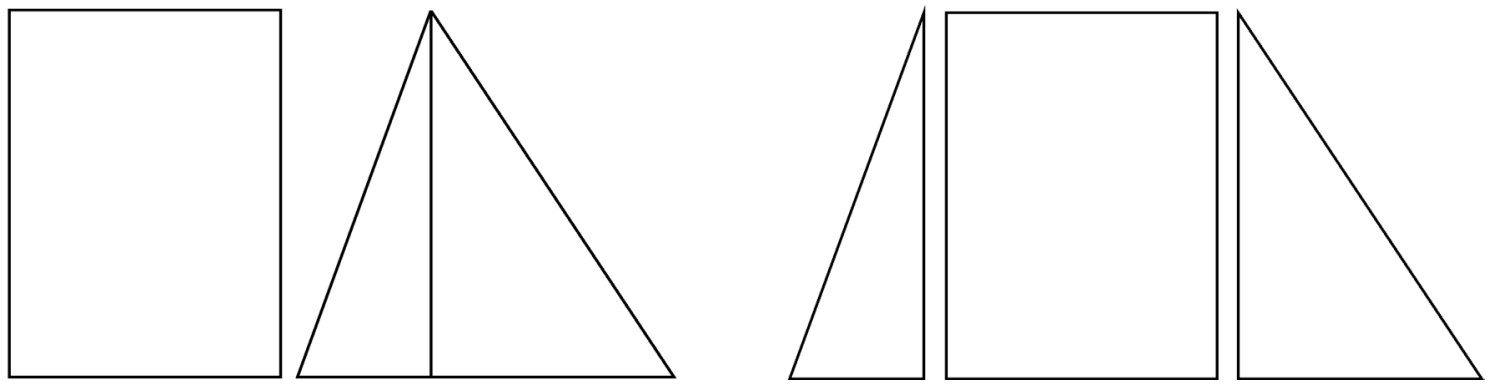

Figura 11a, b. Rappresentazione della scomposizione del trapezio in un rettangolo e in triangoli.

Soluzione 3B. Altri cinque bambini suddividono il trapezio in triangoli, anche in questo caso con due soluzioni diverse (Figure 12a e 12b).
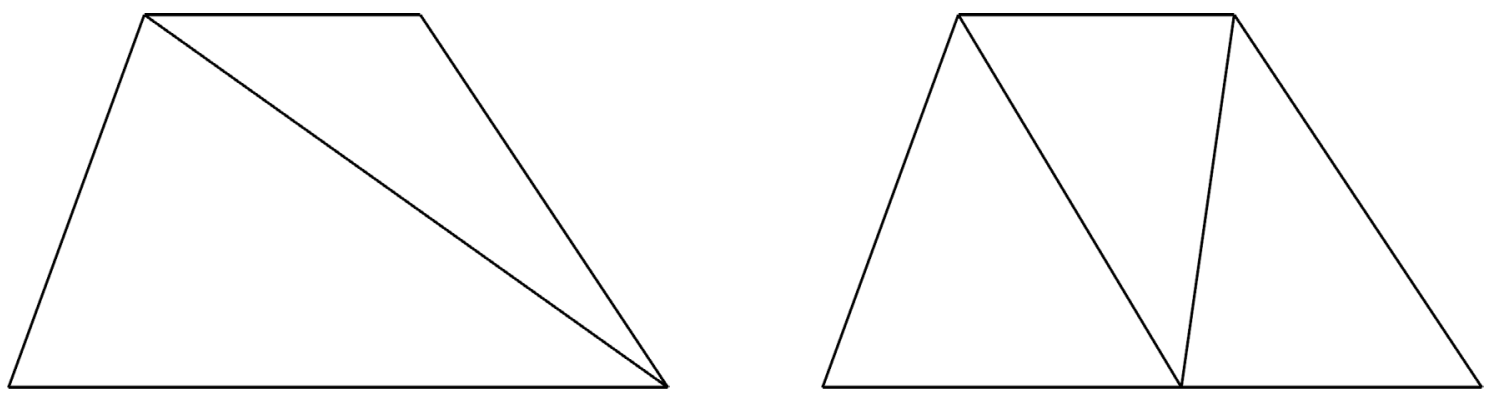

Figura 12a, b. Rappresentazione della scomposizione del trapezio in triangoli.

M2. argomenta così la sua scomposizione (Figura 12a):

«lo ho pensato di dividere il trapezio con la diagonale così formo due triangoli, uno è appogiato sulla base del trapezio, l'altro è capovolto. Se giro il trapezio lo vedo normale, ma non vedo bene l'altezza abbiamo imparato che non va a finire sulla base perché è troppo corta allora devo allungarla oppure la base è la diagonale».

M2. manifesta un disagio che non è relativo al riconoscimento della forma triangolare in una posizione non standard, ma riguarda il punto di vista con cui vedere il triangolo relativamente alla base da prendere in considerazione per tracciare l'altezza.

C. invece pensa a una scomposizione in tre triangoli tipica di una tassellazione (Figura 12b).

Soluzione 3C. Un altro allievo, B., scrive: "Traccio le due diagonali, mi sembrano perpendicolari, assomiglia a un rombo allora posso fare come abbiamo imparato a fare per il rombo, le moltiplico e poi divido per due». L'insegnante gli propone di verificare l'impressione di somiglianza tra il trapezio e il rombo utilizzando le conoscenze relative alle due figure. A questo punto $B$. riconsidera ciò che aveva pensato. Anche lui divide il trapezio in due triangoli (Figura 12a). Questo protocollo richiama l'attenzione su quanto il vedere, separato dal pensiero, possa condurre a fenomeni di cortocircuito: il bambino autore del testo è un allievo generalmente molto acuto, ma che spesso fa troppo affidamento ad argomentazioni di tipo numerico. 


\subsection{Riflessioni sulle soluzioni emerse}

Tutti i bambini sembrano essere giunti a una soluzione del problema. La quasi totalità degli allievi perviene ad un lavoro interno alla figura, quello che Duval chiama la decostruzione dimensionale delle figure, che è un modo di vedere le figure che si possono costruire su carta che è specifico della geometria.

Duval osserva che:

«la decostruzione dimensionale ignora totalmente le dimensioni delle unità figurali. Nel modo matematico di vedere le figure, le misure di grandezza non contano. Detto in altro modo, la visualizzazione geometrica non ha alcun legame con la geometria empirica nella quale il primo gesto è il gesto concreto di misurare lunghezze per fare calcoli utilizzando le formule [...] la vera domanda per un'introduzione della geometria alla primaria non è sapere quali oggetti geometrici scegliere o quali attività di costruzione di figure far fare, ma come prendere coscienza del modo matematico di vedere le figure [...] fin dall'inizio gli studenti devono acquisire la capacità di "uscire dalla figura data" estendendo i lati disegnati e la capacità di aggiungere linee tracciate all'interno della figura per dividerla. [...] Queste attività di ricostruzione sono dei veri problemi da risolvere con strumenti non graduati».

(Duval, 2018, pp. 230-231, corsivo aggiunto dall'autore)

L'attività sul trapezio si è conclusa con un lavoro a gruppi e una discussione. Nel lavoro a gruppi i bambini erano stati divisi per affinità di soluzione e il compito assegnato era di pervenire ad una regola per calcolare l'area del trapezio a partire dalla configurazione realizzata. Questo momento di discussione tra pari in alcuni gruppi è stato molto vivace, soprattutto per la difficoltà di passare dal piano operativo a un piano linguistico geometrico efficace per comunicare una regola. Dal punto di vista del senso del lavoro geometrico, ha dato modo agli allievi di comprendere la genesi di una formula e il suo collegamento con una modalità di vedere la situazione. Anche nel caso del trapezio sono state costruite due formule, dando luogo all'idea che la formula utilizzata (quella che essi trovano sui libri) è una delle possibili, come ha affermato un'allieva probabilmente «quella che è stata giudicata dai matematici più breve, più economica».

Ecco due esempi di conclusioni del lavoro dei gruppi.

Uno dei gruppi che ha lavorato sulle soluzioni 3A (Figura 11a) scrive:

«La regola potrebbe essere base minore del trapezio $x$ altezza + base maggiore - base minore $x$ altezza : 2. Abbiamo ipotizzato che la regola possa essere base minore per altezza, perché la base del rettangolo corrisponde sempre alla base minore del trapezio, $x$ altezza perché l'altezza del rettangolo corrisponde all'altezza del trapezio, poi base maggiore - base minore perché in tutti i casi quando sottrai la base minore dalla base maggiore ti viene la base del triangolo che otteniamo, $x$ altezza perché l'altezza del triangolo corrisponde all'altezza del trapezio, : 2 perché dato che stiamo calcolando l'area del triangolo bisogna sempre dividere per $2 »$.

Il lavoro di questo gruppo riformula con un linguaggio aderente agli elementi del trapezio la strategia che era stata seguita da quegli alunni. Più complessa è la conclusione di uno dei gruppi che ha lavorato sulle soluzioni 3B (Figura 12a):

«La regola che abbiamo trovato e quella di sommare la base maggiore alla base minore, poi moltiplicare il risultato per l'altezza del trapezio. La base maggiore e minore del trapezio sono le basi dei triangoli e l'altezza del trapezio è uguale all'altezza dei triangoli. È come se facessimo un triangolo solo. Poi però visto che otteniamo un rettangolo che è il doppio del trapezio dobbiamo dividere il risultato in due». 
Questo gruppo costruisce la rappresentazione tagliando il trapezio lungo la diagonale e ribaltando un triangolo (Figura 13, disegno originale), col principale scopo di capire il proprio ragionamento, che era partito dal pensare l'area del trapezio come la somma delle aree dei triangoli. Questa volta l'idea di allineare i triangoli dopo il ritaglio provoca la sofferta difficoltà di far coincidere la nuova configurazione con la congettura formulata a causa dell'altezza del triangolo ottusangolo. Nasce una proficua discussione nel gruppo che intreccia l'idea di formulare una regola unica (cioè non la somma di due regole, come la conclusione a cui è giunto il gruppo precedente) e il dover "parlare con le parole del trapezio" pur guardando due triangoli. Questo andare e venire dall'aspetto iconico alla verbalizzazione che seleziona e chiarisce permette loro di giungere alla definizione di un pensiero che vede nella configurazione ottenuta il trapezio originale e il proprio doppio.

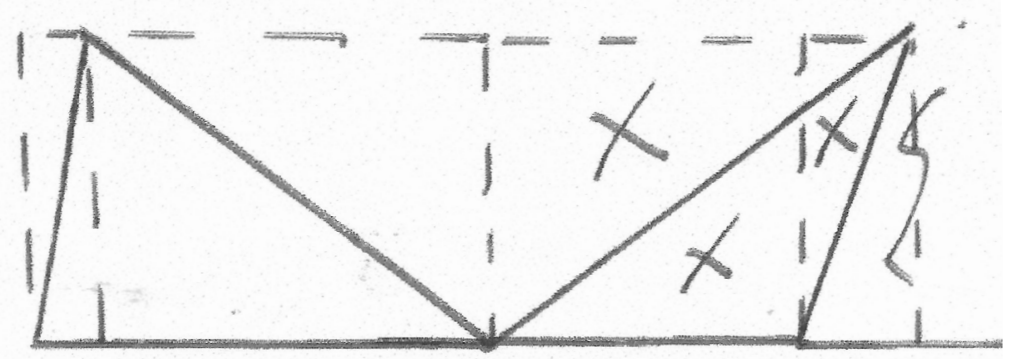

Figura 13. Rappresentazione originale prodotta dal gruppo: è evidente lo sforzo di giungere a capire che l'area del rettangolo avente come base la somma delle basi del trapezio è il doppio di quella del trapezio.

Per un altro gruppo di allievi, che ha lavorato sulle strategie di tipo 3B, partendo dalle soluzioni evidenziate nella Figura 12b, la possibilità di allineare i tre triangoli (Figura 14) ha reso più agevole la comprensione della regola, come riportato dal loro scritto:

"Se mettiamo i tre triangoli uno accanto all'altro e immaginiamo di trovare l'area del trapezio, è come se ogni triangolo fosse la metà di un rettangolo. Tutti e tre insieme sono la metà di un rettangolo che li tiene tutti dentro, Quel rettangolo ha la base che è la somma delle due basi del trapezio e l'altezza uguale a quella del trapezio, solo che il trapezio è rappresentato dai tre triangoli, quindi la sua area sarà la metà di quella del rettangolo e l'altezza del trapezio è uguale all'altezza dei triangoli».

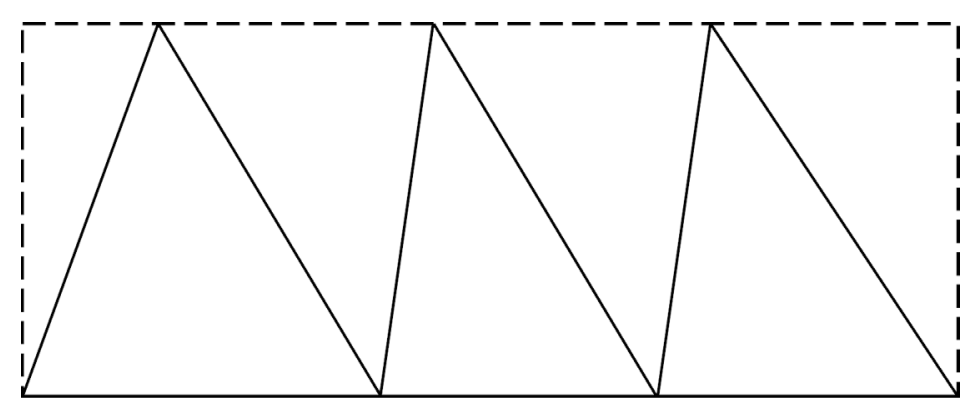

Figura 14. L'area del trapezio è metà dell'area del rettangolo che lo contiene. 


\section{Leprove INvaLSI}

Nel secondo quadrimestre della classe quinta sono stati proposti agli allievi alcuni item delle prove INVALSI somministrate negli anni precedenti riguardanti aspetti geometrici, per avere degli elementi di valutazione su base più ampia. I risultati ottenuti nella classe, confrontati con quelli su scala nazionale, sono risultati sempre più alti.

Riportiamo qui alcuni esempi.

Nella prova di grado 5 del 2016 l'item D19 chiedeva di confrontare due configurazioni indicando se avessero la medesima area (Figura 15). La domanda, a risposta chiusa, prevedeva due opzioni per ciascuna risposta, da scegliere in base alla motivazione dichiarata. L'item era di una certa difficoltà, a causa della funzione dei distrattori: I'opzione A coglie un livello di accostamento unicamente basato sulla percezione; l'opzione $C$ individua chi mette in relazione la lunghezza del perimetro con la dimensione dell'area; I'opzione $\mathrm{D}$, che afferma che le due aree sono uguali, porta una motivazione in sé corretta, ma non pertinente alla domanda. Per scegliere la risposta corretta (B) occorre riconoscere le figure interne disegnate anche in posizioni non standard.

Dai dati INVALSI risulta che su scala nazionale solo il 41,4\% degli allievi ha scelto la risposta corretta e che circa il $50 \%$ degli alunni ha detto che le due configurazioni non avevano la stessa area, scegliendo le risposte $\mathrm{A}$ e $\mathrm{C}$.

La percentuale di risposte corrette nella classe oggetto di questa sperimentazione è stata del $77,2 \%$, tenendo conto di tutti gli alunni, compresi i tre allievi con certificazione di disabilità e i due bambini con DSA che I'INVALSI non include nelle percentuali presentate. Nonostante il risultato possa essere interpretato come globalmente positivo, è da rilevare che 3 allievi hanno scelto I'opzione A e 1 allievo l'opzione C.

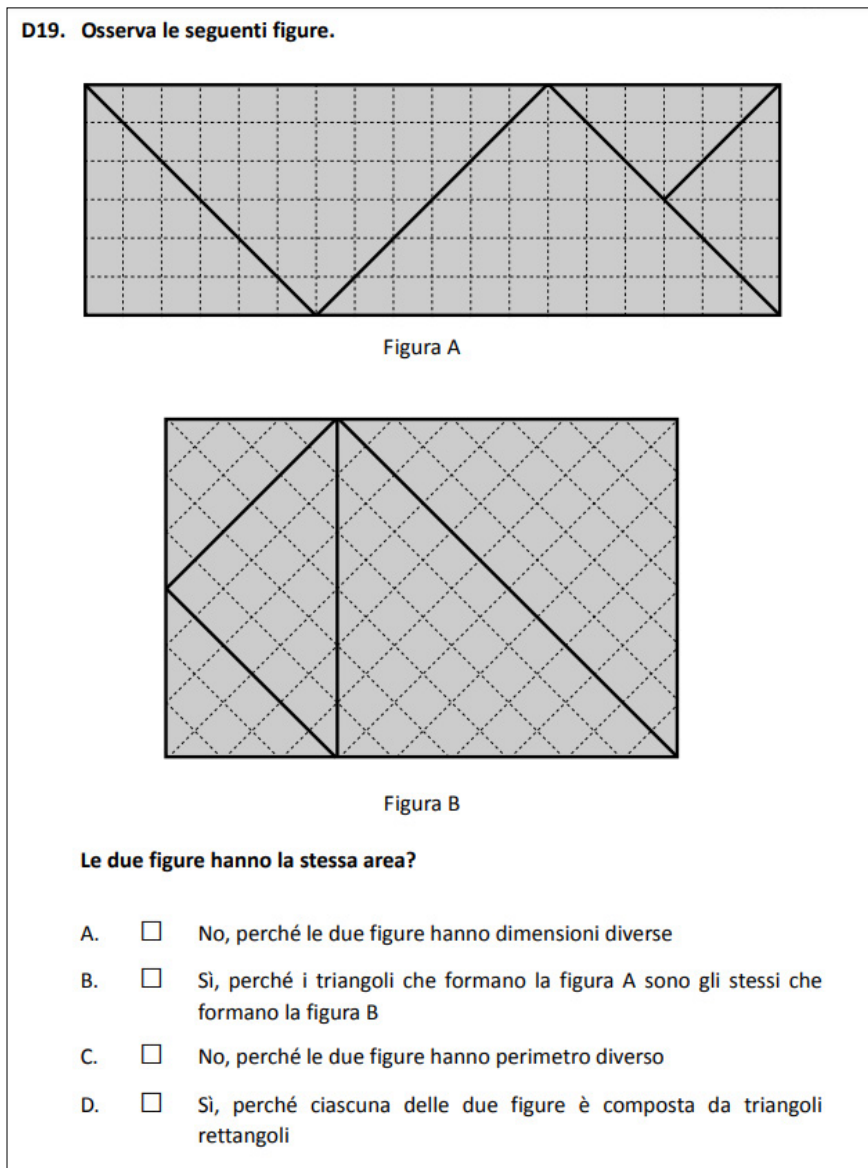

Figura 15. Domanda D19 della prova INVALSI di matematica di grado 5 del 2016. 
L'item seguente (Figura 16), invece, è tratto dalla prova svolta dalla classe stessa a maggio, prova svolta in modo rigoroso con un insegnante esterno al team e all'ambito matematico. L'item entra nel merito delle problematiche della visualizzazione, anche se si presenta come una situazione abbastanza semplice. II dato nazionale è particolarmente alto: $81,2 \%$ di risposte corrette. La classe ha risposto positivamente al 100\%: sul piano della relazione con la visione anticipativa il lavoro svolto durante I'anno sembra aver costruito le abilità di base in tutti i bambini (anche in questo dato sono compresi i bambini con difficoltà di apprendimento).

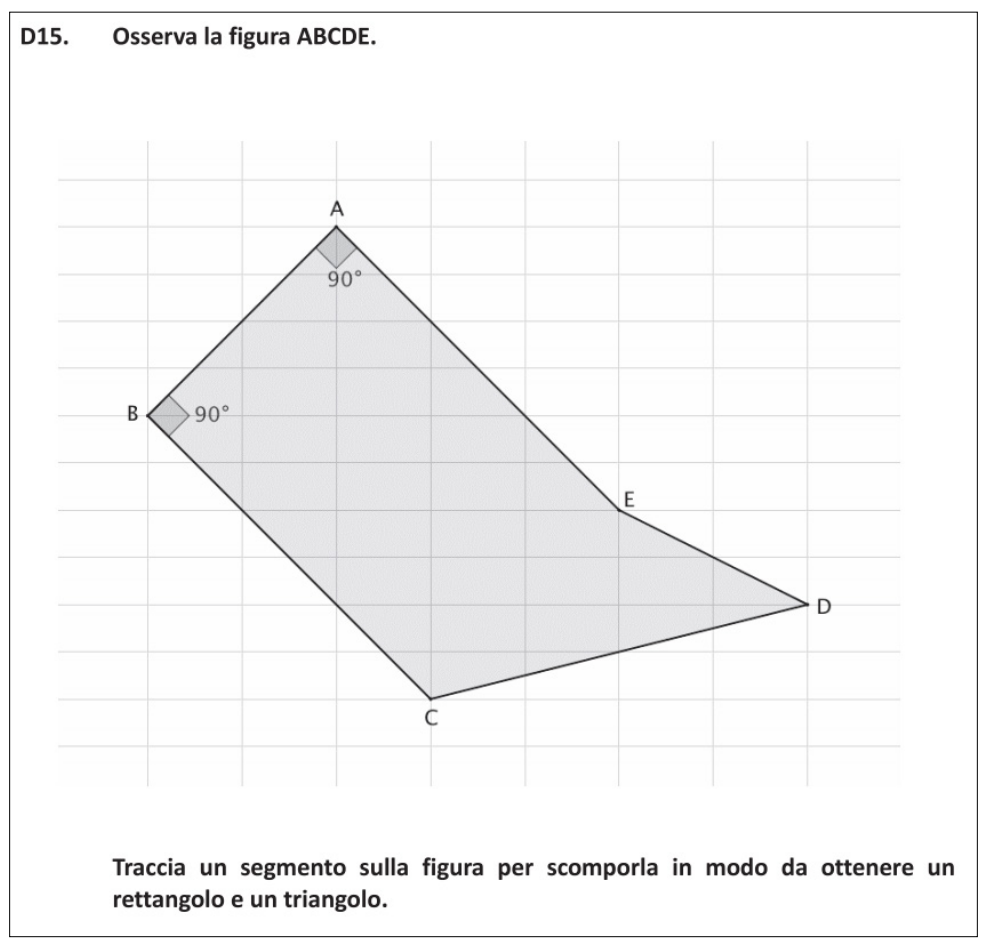

Figura 16. Domanda D15 della prova INVALSI di matematica di grado 5 del 2018

Nella seconda metà dell'anno, inoltre, la classe ha effettuato, all'interno del progetto di tesi di una studentessa di Scienze della Formazione Primaria, un'esperienza di geometria dinamica su GeoGebra, in cui è stato richiesta, in un ambiente diverso, la messa in gioco di atteggiamenti esplorativi non lontani da quelli descritti in questo articolo (si veda Soldano, Sabena \& Scali, 2018).

\section{Conclusioni}

Nell'esperienza condotta si è cercato di progettare delle occasioni di lavoro geometrico nel senso indicato da Duval (2018).

I risultati non sono stati raccolti da un punto di vista quantitativo, ma nel corso dell'articolo sono stati indicati diversi aspetti qualitativi utili all'insegnante per monitorare in itinere il lavoro svolto. II momento della discussione, inteso come discussione su un oggetto matematico orchestrata dall'insegnante ha costituito la possibilità per molti bambini da un lato, di prendere coscienza di aspetti di cui erano inconsapevoli riguardanti la propria soluzione, dall'altro di poter fare esperienza di modi diversi di visualizzare le configurazioni, dall'altro ancora di poter essere esposti a processi di andata-ritorno 
tra le figure e gli aspetti analitico-concettuali.

Molti allievi hanno mostrato un significativo cambiamento nelle proprie strategie, in una direzione di maggior maturità nell'accostamento ad un modo geometrico di pensare alle figure. Tutti hanno partecipato in modo attivo ai momenti di lavoro collettivo, che si sono prolungati spesso oltre i tempi prefissati. Come inciso, va detto che alcuni dei testi e degli interventi riportati nel corso dell'articolo sono stati prodotti da allievi con bisogni educativi speciali:4 il fatto che sia difficile distinguere le loro produzioni tra tutte quelle proposte mostra come il lavoro svolto con attenzione allo sviluppo delle capacità di verbalizzazione degli allievi sia stato efficace negli anni e abbia supportato adeguatamente anche questa esperienza didattica.

È parsa significativa, in particolare, la possibilità di verbalizzare ciò che la visualizzazione suggeriva: in questo senso ogni figura parla e la verbalizzazione chiarisce e dà voce al pensiero generato dal vedere la figura. Questo ha comportato, ad esempio, la necessità di adattare il vocabolario geometrico alle diverse configurazioni realizzate, accostando di volta in volta il termine che designava ciò che si stava vedendo: nel caso del rombo, ad esempio, la base di uno dei triangoli in cui era stato scomposto corrispondeva alla diagonale del rombo.

Talvolta, tuttavia, la figura, per qualche allievo, rimane silenziosa e sembra che nella mente si sovrappongano altri pensieri, da cui hanno origine dei cortocircuiti. Succede anche in presenza di una didattica attenta a non valorizzare i risultati a scapito dei processi.

Questo, tuttavia, anziché demotivare, incentiva il docente a proseguire nella ricerca di modalità sempre più efficaci di costruzione di abilità e competenze geometriche.

\section{Bibliografia}

Baccaglini Frank, A., Di Martino, P., Natalini, R., Rosolini, G. (2018). Didattica della matematica, Mondadori.

Bussi, M. B., Boni, M., \& Ferri, F. (1995). Interazione sociale e conoscenza a scuola: la discussione matematica, rapporto tecnico N 21-Nucleo di ricerca in Storia e Didattica della Matematica Pura ed Applicata. Università degli Studi di Modena, Centro Documentazione Educativa Comune di Modena-Settore Istruzione, 11-13.

Boero, P. (1990). L'insegnamento della matematica nel progetto "Bambini, Maestri, Realtà". L'insegnamento della matematica e delle scienze integrate, 13, 7-43.

Boero, P., Dapueto, C., Ferrari, P., Ferrero, E., Garuti, R., Lemut, E., Parenti, L., \& Scali, E. (1995). Aspects of the Mathematics-Culture Relationship in mathematics teaching-learning in compulsory school. PME conference proceedings Recife, Brasil, 151-166.

Douady, R. (1986). Jeux de cadres et dialectique outil-objet. Recherches en didactique des mathématiques, 7(2), 5-31.

Duval, R. (2018). Per l'educazione allo sguardo in geometria elementare e in pittura. La matematica e la sua didattica, 26(2), 211-245.

Fischbein, E. (1992). Intuizione e dimostrazione. In B. D'Amore (A cura di), Matematica a scuola (pp. 1-24). Bologna: Pitagora.

Fischbein, E. (1993). The theory of figural concepts. Educational Studies in Mathematics, 24(2), 139-162.

4. Hanno lavorato su questa esperienza due allievi con una certificazione di un lieve ritardo mentale e un terzo con serie problematiche nella sfera emotiva, e un allievo con la diagnosi di funzionamento cognitivo al limite. 
Laborde, C. (1988). L'enseignement de la géométrie en tant que terrain d'exploration de phénomènes didactiques, Recherches en didactique des mathématiques, 9(3), 337-364.

Mariotti, A. (2005). La geometria in classe. Riflessioni sull'insegnamento e l'apprendimento della geometria. Bologna: Pitagora.

MIUR (2012). Indicazioni Nazionali per il curricolo della scuola dell'infanzia e del primo ciclo di istruzione. Annali della Pubblica Istruzione, Numero speciale. Firenze: Le Monnier.

Parzysz, B. (1988) Knowing vs Seeing. Problems of the plane representation of space geometry figures, Educational Studies in Mathematics, 19(1), 79-92.

Pellerey, M. (2004). Le competenze individuali e il portfolio. Milano: La Nuova Italia.

Sabena, C., Ferri, F., Martignone, F., \& Robotti, E. (2019). Insegnare e apprendere matematica nella scuola dell'infanzia e primaria. Mondadori Università.

Soldano, C., Sabena, C., \& Scali, E. (2018). L'area dei quadrilateri: attività di gioco e indagine geometrica con GeoGebra, XXXV Convegno UMI-CIIM, Cagliari, 4-6 ottobre 2018.

Vygotskij, L. S. (1990). Pensiero e linguaggio, a cura di L. Mecacci, Bari: Laterza (Titolo originale Myšlenie i reč: Psicholodičeskie issledovanija pubblicato nel 1934).

Wertheimer, M. (1997). I/ pensiero produttivo. Milano: Giunti.

Zan, R. (2007). Difficoltà in matematica. Osservare, interpretare, intervenire. Milano: Springer

Zan, R, (2016). I problemi di matematica: difficoltà di comprensione e formulazione del testo. Roma: Carocci Faber. 